\title{
Cyclic Presentations of the Trivial Group
}

\author{
Martin Edjvet, Paul Hammond, and Nathan Thomas
}

\section{CONTENTS}

1. Introduction

2. The Experiment

3. Results

References
Hammond was supported by EPSRC Postgraduate Studentship Award No. 97004151.
We report on a computational group theory experiment involving a search for cyclic presentations of the trivial group. The list of such presentations obtained includes counterexamples to a conjecture of M J Dunwoody.

\section{INTRODUCTION}

We report on a computational group theory experiment, involving the use of QUOTPIC [Holt and Rees 1993] and designed to test one of two conjectures made by M. J. Dunwoody [1995] on cyclically presented groups. (The other conjecture, not considered here, has been proved independently in [Cavicchioli et al. 1999] and [Song and Kim 1999]. Roughly it stated that any closed orientable 3-manifold represented by a symmetric Heegaard diagram is homeomorphic to a cyclic covering of the 3 -sphere branched over some knot.)

Let $F_{n}$ denote the free group on $n$ (free) generators $x_{0}, \ldots, x_{n-1}$ and let $\theta: F_{n} \rightarrow F_{n}$ be the automorphism for which $x_{i} \theta=x_{i+1}$ (where subscripts are taken modulo $n$ ). Following [Johnson 1980], for (cyclically reduced) $w \in F_{n}$ define $G_{n}(w)=$ $F_{n} / N$ where $N$ is the normal closure in $F_{n}$ of the set $\left\{w, w \theta, \ldots, w \theta^{n-1}\right\}$. A group $G$ is said to have a cyclic presentation or to be cyclically presented if $G \cong G_{n}(w)$ for some $w$ and for some $n$. The polynomial associated with the cyclic presentation for $G_{n}(w)$ is defined to be $f_{w}(t)=\sum_{i=0}^{n-1} a_{i} t^{i}$ where $a_{i}$ is the exponent sum of $x_{i}$ in $w$. Put $A_{n}(w)=$ $G_{n}(w)^{a b}$. It is shown in [Johnson 1980] that the order of $A_{n}(w)$ is equal to the absolute value of the product $\prod_{i=0}^{n-1} f_{w}\left(\xi_{i}\right)$ where $\xi_{i}$ ranges over the set of complex $n$th roots of unity (with the convention that $A_{n}(w)$ is infinite whenever the product vanishes). Furthermore $A_{n}(w)$ is trivial if and only if $f_{w}(t)$ is a unit in the ring $\mathbb{Z}[t] /\left(t^{n}-1\right)$. 
Dunwoody [1995] conjectured that if $G_{n}(w)$ is trivial then $f_{w}(t)= \pm t^{i}$. If $n=2,3,4$, or 6 the only units in $\mathbb{Z}[t] /\left(t^{n}-1\right)$ are cosets containing elements of the form $\pm t^{i}$, so it follows from the discussion above that the conjecture is true for these values of $n$. As a result of our experiment we can report that the conjecture is false for $n=5$, a counterexample being $G_{5}\left(x_{0}^{-1} x_{1}^{-1} x_{3} x_{2} x_{1}\right)$.

In fact our initial impetus was to find examples of trivial $G_{n}(w)$ where $n \geq 4$ and where the presentation is irreducible (see Section 2). One motivation being that any such presentation could be a possible counterexample to the well-known AndrewsCurtis conjecture (see [Burns and Macedońska 1993; Baumslag et al. 1999] for a discussion of this).

In Section 2 we describe the experiment and in Section 3 we list the results achieved so far.

\section{THE EXPERIMENT}

Recall that we are searching for possible $w$ such that $G_{n}(w)$ is trivial.

Firstly we will insist that the presentation for $G_{n}(w)$ is irreducible, that is, if $w$ involves $x_{i_{1}}, \ldots, x_{i_{k}}$ only, where $i_{j}<i_{j+1}$, then $h c f\left(i_{2}-i_{1}, i_{3}-i_{2}, \ldots, i_{k}-\right.$ $\left.i_{k-1}, n\right)=1$. This will prevent the group $G_{n}(w)$ decomposing into a free product of copies of $G_{m}(\hat{w})$ where $m$ divides $n$. As an illustration consider

$$
G_{9}\left(x_{0}^{-1} x_{3} x_{0} x_{3}^{-2}\right) \text {. }
$$

This group can be expressed as the free product

$$
\begin{aligned}
& \left\langle x_{0}, x_{3}, x_{6} \mid x_{0}^{-1} x_{3} x_{0} x_{3}^{-2}, x_{3}^{-1} x_{6} x_{3} x_{6}^{-2}, x_{6}^{-1} x_{0} x_{6} x_{0}^{-2}\right\rangle * \\
& \left\langle x_{1}, x_{4}, x_{7} \mid x_{1}^{-1} x_{4} x_{1} x_{4}^{-2}, x_{4}^{-1} x_{7} x_{4} x_{7}^{-2}, x_{7}^{-1} x_{1} x_{7} x_{1}^{-2}\right\rangle * \\
& \left\langle x_{2}, x_{5}, x_{8} \mid x_{2}^{-1} x_{5} x_{2} x_{5}^{-2}, x_{5}^{-1} x_{8} x_{5} x_{8}^{-2}, x_{8}^{-1} x_{2} x_{8} x_{2}^{-2}\right\rangle
\end{aligned}
$$

and each of the (isomorphic) free factors are known to be trivial [Higman 1951]. After renumbering, each free factor is $G_{3}\left(x_{0}^{-1} x_{1} x_{0} x_{1}^{-2}\right)$. Observe also that the associated polynomial of $G_{n}(w)$ is $\pm t^{i}$ for some $i$ if and only if the associated polynomial of $G_{m}(\hat{w})$ is $\pm t^{j}$ for some $j$.

Now the automorphism $\theta$ of the free group $F_{n}$ defined in Section 1 induces an automorphism of $G_{n}(w)$ of order dividing $n$ and the resulting split extension $H_{n}(w)$ of $G_{n}(w)$ by the cyclic group of order $n$ has a presentation

$$
H_{n}(w)=\left\langle x, t \mid t^{n}, w(x, t)\right\rangle
$$

where $w(x, t)$ is in the normal closure of $x$ and $t^{n}$. (See [Johnson 1980], for example.) Conversely any group with such a presentation is a split extension of a $G_{n}(w)$ for some $w$. For example, if $n=4$ and $w=x_{0} x_{1} x_{3}^{-1} x_{1}^{-1} x_{3}$ then

$$
H_{n}(w)=\left\langle x, t \mid t^{4}, x t^{-1} x t^{-2} x^{-1} t^{2} x^{-1} t^{-2} x t^{3}\right\rangle .
$$

This observation provides us with the parameters we shall use, namely $n$ and $l=l(w(x, t))$, that is, $n$ together with the length of the word $w(x, t)$ regarded as an element in the free group on $x$ and $t$ (so in the above example, $l=15$ ).

In fact we make the following assumptions:

$$
4 \leq n \leq 10 \quad \text { and } \quad l=l(w(x, t)) \leq 15 .
$$

This, in principle, means that for each $n$ there are $2\left(3^{15}-1\right)$ reduced $w(x, t)$ to consider, but of course there are further restrictions we can make and now list.

1. The word $w(x, t)$ is cyclically reduced.

2. The exponent sum of $t$ in $w(x, t)$ is congruent to 0 modulo $n$.

3. We can work modulo equivalence where $w_{1}(x, t)$ is equivalent to $w_{2}(x, t)$ if and only if $w_{1}(x, t)$ can be obtained from $w_{2}(x, t)$ by a sequence of the following moves:

(a) cyclic permutation;

(b) replace $x$ by $x^{-1}$ everywhere;

(c) replace $t$ by $t^{-1}$ everywhere;

(d) inversion.

4. The exponent sum of $x$ in $w(x, t)$ is equal to 1 .

5. No cyclic permutation of $w(x, t)$ contains the subwords $t^{-k}, t^{k+1}$ (if $n=2 k$ ) or $t^{-(k+1)}, t^{k+1}$ (if $n=2 k+1)$.

This completes our restrictions for $w(x, t)$, and we have produced a computer programme that lists all the resulting $w(x, t)$. The programme then rewrites each $w(x, t)$ into a word $w$ in the $x_{i}(0 \leq i \leq n-1)$ and at this stage there are three further restrictions we can make.

6. The resulting presentation is irreducible in the sense discussed at the beginning of this section.

7. The determinant of the relation matrix of the resulting presentation equals \pm 1 .

8. The word $w$ involves at least three of the $x_{i}$.

This last restriction follows from Theorem 3 in [Pride 1987]. Part of this theorem implies that if 
$w$ involves $x_{0}$ and $x_{1}$ only and $n \geq 4$ then $G_{n}(w)$ is nontrivial. Since we are dealing with irreducible presentations it can be assumed that if $w$ involves only two of the $x_{i}$ then (after renumbering, if necessary) they are $x_{0}$ and $x_{1}$.

This way we end up with a list of words $w$ that are candidates for $G_{n}(w)$ to be trivial. We have used QUOTPIC to investigate some of these presentations. If we can find any proper subgroups of finite index, for example, then the corresponding $w$ can be discarded from our list; likewise if we can find a nontrivial quotient of $G_{n}(w)$. (The interested reader wanting further details is invited to contact the first named author.) Finally, when we could not show that a particular $G_{n}(w)$ is nontrivial we used the coset enumeration programme in QUOTPIC relative to the trivial subgroup to check for triviality and in some cases found the order of $G_{n}(w)$ to equal 1 (of course in many cases we obtained no information in that the coset enumeration did not complete).

\section{RESULTS}

After applying our first programme, before using QUOTPIC, we found that the number of candidates $w$ for which $G_{n}(w)$ might be trivial is given for each $n$ in Table 1, where we have partitioned the words $w$ into those that yield a presentation whose associated polynomial is $\pm t^{i}$ and otherwise. Thus the words after the / in each entry of Table 1 represent possible counterexamples to Dunwoody's conjecture.

\begin{tabular}{|c|c|c|c|c|c|c|c|}
\hline$l$ & $n=4$ & $n=$ & $5 \quad n=6$ & $n=7$ & $n=8$ & $n=9$ & $n=10$ \\
\hline 7 & $0 / 0$ & $0 /$ & $0 / 0$ & $0 /$ & $0 / 0$ & $0 / 0$ & $0 / 0$ \\
\hline 8 & $0 / 0$ & $0 /$ & $0 / 0$ & $0 / 0$ & $0 / 0$ & $0 / 0$ & $0 / 0$ \\
\hline 9 & $0 / 0$ & $0 /$ & $0 / 0$ & $0 / \quad 1$ & $0 / 0$ & $0 /$ & $0 / 0$ \\
\hline 10 & $0 / 0$ & $0 / \quad$ & $0 / 0$ & $0 / \quad 2$ & $0 /($ & $0 / 0$ & $0 / 0$ \\
\hline 11 & $4 / 0$ & $4 /$ & $4 / 0$ & $4 / 2$ & $4 / 0$ & $4 / 0$ & $4 / 0$ \\
\hline 12 & $0 / 0$ & $3 /$ & $0 / 0$ & $0 / 3$ & $0 / 0$ & $0 / 0$ & $0 / 0$ \\
\hline 13 & $17 / 0$ & $17 / 19$ & $921 / 0$ & $21 / 21$ & $21 / 0$ & $21 / 3$ & $21 / 2$ \\
\hline 14 & $0 / 0$ & $8 / 26$ & $0 / 0$ & $3 / 14$ & $0 / 0$ & $0 / 2$ & $0 / 0$ \\
\hline 15 & $103 / 0$ & $93 / 11$ & $\begin{array}{lll}3 & 105\end{array}$ & $105 / 103$ & 0 & $09 / 9$ & $09 / 11$ \\
\hline
\end{tabular}

TABLE 1. Number of candidate words $w$ for which $G_{n}(w)$ might be trivial, after first phase; $x / y$ indicates the are $x$ such words that yield a presentation whose associated polynomial is $\pm t^{i}$, and $y$ that don't.
Since $x$ has exponent sum 1 in $w(x, t)$ each $w$ in Table 1 can be put into the form $x_{i} w^{\prime}$ where $w^{\prime}$ is in the commutator subgroup. It follows that if $4 \leq n \leq 10$ and $l \leq 14$ then $w$ is one of

$$
\begin{array}{lc}
x_{2}\left[x_{1}^{\varepsilon_{1}}, x_{0}^{\varepsilon_{2}}\right] & (4 \leq n \leq 10, l=11) \\
x_{3}\left[x_{1}^{\varepsilon_{1}}, x_{0}^{\varepsilon_{2}}\right] & \left(n=5, l=12,\left(\varepsilon_{1}, \varepsilon_{2}\right) \neq(-1,-1)\right) \\
& \quad(6 \leq n \leq 10, l=13) \\
x_{2}\left[x_{1}^{\varepsilon_{1}}, x_{0}^{2 \varepsilon_{2}}\right] & (4 \leq n \leq 10, l=13) \\
x_{2}\left[x_{1}^{2 \varepsilon_{1}}, x_{0}^{\varepsilon_{2}}\right] & (4 \leq n \leq 10, l=13) \\
x_{2}\left[x_{0}^{\varepsilon_{1}} x_{1}^{\varepsilon_{2}}, x_{1}^{\varepsilon_{2}}\right] & (4 \leq n \leq 10, l=13) \\
x_{2}\left[x_{1}^{\varepsilon_{1}}, x_{0}\right]\left[x_{0}, x_{1}^{-\varepsilon_{1}}\right] & (4 \leq n \leq 10, l=13) \\
x_{2}\left[x_{1}^{\varepsilon_{1}}, x_{3}^{\varepsilon_{2}}\right] & (4 \leq n \leq 10, l=13, \\
& \left.\left(\varepsilon_{1}, \varepsilon_{2}\right) \neq(-1,-1)\right) \\
x_{3}\left[x_{1}^{\varepsilon_{1}}, x_{0}^{2 \varepsilon_{2}}\right] & (n=5, l=14) \\
x_{3}\left[x_{2}^{\varepsilon_{1}}, x_{0}^{\varepsilon_{2}}\right] & (n=5, l=14)
\end{array}
$$

where $\varepsilon_{i}= \pm 1$.

As a result of our use of QUOTPIC we found the following examples of cyclic presentations of the trivial group.

$\begin{array}{lll}G_{4}\left(x_{2} x_{1} x_{0} x_{1}^{-1} x_{0}^{-1}\right) & f_{w}(t)=t^{2} & l=11 \\ G_{4}\left(x_{2} x_{1}^{-1} x_{3}^{-1} x_{1} x_{3}\right) & f_{w}(t)=t^{2} & l=13 \\ G_{4}\left(x_{3} x_{2} x_{1} x_{0}^{-1} x_{1}^{-1} x_{2}^{-1} x_{0}\right) & f_{w}(t)=t^{3} & l=15 \\ G_{4}\left(x_{3} x_{0}^{-1} x_{1} x_{2} x_{1}^{-1} x_{2}^{-1} x_{0}\right) & f_{w}(t)=t^{3} & l=15 \\ G_{4}\left(x_{3} x_{2} x_{1} x_{0}^{-1} x_{1}^{-1} x_{0} x_{2}^{-1}\right) & f_{w}(t)=t^{3} & l=15 \\ G_{4}\left(x_{3} x_{0}^{-1} x_{1} x_{2} x_{1}^{-1} x_{0} x_{2}^{-1}\right) & f_{w}(t)=t^{3} & l=15 \\ G_{5}\left(x_{0}^{-1} x_{1}^{-1} x_{3} x_{2} x_{1}\right) & f_{w}(t)=-1+t^{2}+t^{3} & l=11 \\ G_{5}\left(x_{0}^{-1} x_{2} x_{3}^{-1} x_{0} x_{4}\right) & f_{w}(t)=t^{2}-t^{3}+t^{4} & l=12 \\ G_{5}\left(x_{0}^{-1} x_{2} x_{1}^{-1} x_{3} x_{1}\right) & f_{w}(t)=-1+t^{2}+t^{3} & l=13 \\ G_{5}\left(x_{0}^{-1} x_{2}^{-1} x_{0} x_{3} x_{1}\right) & f_{w}(t)=t-t^{2}+t^{3} & l=14\end{array}$

The use of QUOTPIC also allowed us to reduce the number of $w$ for which it is unknown whether $G_{n}(w)$ is trivial. The present totals are given in Table 2 .

\begin{tabular}{|rrrrrrrr|}
\hline$l$ & $n=4$ & $n=5$ & $n=6$ & $n=7$ & $n=8$ & $n=9$ & $n=10$ \\
\hline$\leq 10$ & $0 / 0$ & $0 / 0$ & $0 / 0$ & $0 / 0$ & $0 / 0$ & $0 / 0$ & $0 / 0$ \\
11 & $3 / 0$ & $2 / 3$ & $0 / 0$ & $4 / 0$ & $4 / 0$ & $1 / 0$ & $2 / 0$ \\
12 & $0 / 0$ & $1 / 2$ & $0 / 0$ & $0 / 0$ & $0 / 0$ & $0 / 0$ & $0 / 0$ \\
13 & $3 / 0$ & $8 / 11$ & $2 / 0$ & $17 / 14$ & $4 / 0$ & $9 / 1$ & $6 / 0$ \\
14 & $0 / 0$ & $3 / 14$ & $0 / 0$ & $0 / 9$ & $0 / 0$ & $0 / 0$ & $0 / 0$ \\
15 & $41 / 0$ & $50 / 71$ & $14 / 0$ & $63 / 71$ & $12 / 0$ & $41 / 4$ & $24 / 1$ \\
\hline
\end{tabular}

TABLE 2. Final number of candidate words $w$ for which it is unknown whether $G_{n}(w)$ is trivial; see Table 1 for / convention. 
For example, the $G_{n}(w)$ that remain undecided and where $l(w) \leq 12$ are

$$
\begin{array}{ll}
G_{5}\left(x_{0}^{-1} x_{1}^{-1} x_{2} x_{3} x_{1}\right), & G_{n_{1}}\left(x_{2} x_{1}^{-1} x_{0}^{-1} x_{1} x_{0}\right), \\
G_{5}\left(x_{0}^{-1} x_{1}^{-1} x_{4} x_{1}^{2}\right), & G_{n_{2}}\left(x_{2} x_{1}^{-1} x_{0} x_{1} x_{0}^{-1}\right), \\
G_{5}\left(x_{0}^{-1} x_{2}^{-1} x_{3} x_{2}^{2}\right), & G_{n_{2}}\left(x_{2} x_{1} x_{0}^{-1} x_{1}^{-1} x_{0}\right), \\
G_{5}\left(x_{0}^{-1} x_{2}^{-1} x_{3} x_{0} x_{1}\right), & G_{n_{3}}\left(x_{2} x_{1} x_{0} x_{1}^{-1} x_{0}^{-1}\right), \\
G_{5}\left(x_{0}^{-1} x_{2} x_{4}^{-1} x_{0} x_{1}\right), & G_{5}\left(x_{3} x_{1}^{-1} x_{0}^{-1} x_{1} x_{0}\right),
\end{array}
$$

where $n_{1} \in\{4,7,8\}, n_{2} \in\{4,5,7,8,10\}$, and $n_{3} \in$ $\{7,8,9\}$.

We finish with three remarks. Firstly, in most of our trivial examples $w$ conjugates an element of length $k$ to an element of length $k+1$ (as in Higman's examples). Secondly, in the course of the experiment we discovered some nontrivial finite examples. Curiously, every such example turned out to be isomorphic to $\mathrm{SL}(2,5)$. Finally, the fact that we have not found many examples of irreducible cyclic presentations of the trivial group motivates us to pose the following two questions.

Question 1. Is there an irreducible cyclic presentation of the trivial group with more than 5 generators?

Question 2. Is there an example $w$ where $G_{5}(w)$ is trivial and $f_{w}(t)= \pm t^{i}$ ?

\section{REFERENCES}

[Baumslag et al. 1999] G. Baumslag, A. D. Myasnikov, A. G. Myasnikov, and V. Shpilrain, "On the AndrewsCurtis equivalence", preprint, 1999. Author can be reached at gilbert@groups.sci.ccny.cuny.edu.
[Burns and Macedońska 1993] R. G. Burns and O. Macedońska, "Balanced presentations of the trivial group", Bull. London Math. Soc. 25:6 (1993), 513526.

[Cavicchioli et al. 1999] A. Cavicchioli, B. Ruini, and F. Spaggiari, "On a conjecture of M. J. Dunwoody", preprint, 1999. Author can be reached at alberto@unimo.it.

[Dunwoody 1995] M. J. Dunwoody, "Cyclic presentations and 3-manifolds", pp. 47-55 in Groups - Korea '94 (Pusan, 1994), edited by A. C. Kim and D. L. Johnson, de Gruyter, Berlin, 1995.

[Higman 1951] G. Higman, "A finitely generated infinite simple group", J. London Math. Soc. 26 (1951), 6164 .

[Holt and Rees 1993] D. F. Holt and S. Rees, "A graphics system for displaying finite quotients of finitely presented groups", pp. 113-126 in Groups and computation (New Brunswick, NJ, 1991), edited by L. Finkelstein and W. M. Kantor, DIMACS Ser. Disc. Math. Theoret. Comput. Sci. 11, Amer. Math. Soc., Providence, RI, 1993.

[Johnson 1980] D. L. Johnson, Topics in the theory of group presentations, London Math. Soc. Lecture Note Series 42, Cambridge University Press, Cambridge, 1980.

[Pride 1987] S. J. Pride, "Groups with presentations in which each defining relator involves exactly two generators", J. London Math. Soc. (2) 36:2 (1987), $245-256$.

[Song and Kim 1999] H. J. Song and S. H. Kim, "Dunwoody 3-manifolds and (1,1)-decomposable knots", preprint, 1999. Author can be reached at hjsong@ dolphin.pknu.ac.kr.

Martin Edjvet, School of Mathematical Sciences, University of Nottingham, University Park, Nottingham NG7 2RD, United Kingdom (martin.edjvet@nottingham.ac.uk)

Paul Hammond, School of Mathematical Sciences, University of Nottingham, University Park, Nottingham NG7 2RD, United Kingdom

Nathan Thomas, School of Mathematical Sciences, University of Nottingham, University Park, Nottingham NG7 2RD, United Kingdom

Received May 15, 2000; accepted in revised form August 22, 2000 